\title{
Fundamentals of nanomedicines towards clinical translation
}

\author{
Bruno Sarmento ${ }^{1,2} \cdot$ Steve Little ${ }^{3}$
}

Published online: 3 May 2020

(C) Controlled Release Society 2020

A particular interest in modern translational medicine is nanomedicine, which has opened multiple new doors in the field of drug delivery and continues to be an extremely popular research focus area throughout the world. Specifically, nanomedicine promises to one day allow for site-and-cellspecific targeting, increased absorbability, and even the ability to mimic the action of nano-scale biological features like proteins that exist on the order of one billionth of a meter. Nanomedicine industry is projected to reach a total market value of approximately US $\$ 334$ billion by 2025 [1]. The major portion of nanomedicine market revenue is for therapeutics, consisting of drugs, devices, and drug delivery systems. Seeing this tremendous potential, the Board of Directors of the Controlled Release Society recently prioritized the formation of a Nanomedicine and Nanoscale Delivery (NND) Focus Group [2], which currently is the largest Focus Group in the Society with members from around the globe.

Following an invitation from the Editor in Chief of Drug Delivery and Translational Medicine, Prof Maria José Alonso, we challenged the members of the Nanomedicine and Nanoscale Delivery Focus Group to contribute to the first special edition entitled, "Fundamentals of nanomedicines towards clinical translation." In this issue, we received submissions ranging from the critical thoughts on challenges that need to be considered to promote the clinical translation of nanomedicines to the solid contributions of nanotechnology to the cancer

Bruno Sarmento

bruno.sarmento@ineb.up.pt

Steve Little

srlittle@pitt.edu

1 i3S - Instituto de Investigação e Inovação em Saúde \& INEB Instituto de Engenharia Biomédica, Universidade do Porto, Porto, Portugal

2 Instituto de Investigação e Formação Avançada em Ciências e Tecnologias da Saúde, CESPU, Gandra, Portugal

3 Department of Chemical and Petroleum Engineering, University of Pittsburgh, Pittsburgh, USA therapeutics and diagnostics. These are three valuable contributions from the actual Board of the NNF Focus Group, Mansoor Amiji and collaborators and Twan Lammers and collaborators. The Special Issue also contains two apprised and up-to-dated reviews, one on the importance of perform protein corona studies of polymeric nanoparticles, from Maria José Alonso's group, and a prestigious contribution from Bob Langer and $2019 \mathrm{~T}$. Nagai Postdoctoral Awardee Michael Mitchell on cyclodextrin in delivery systems.

More concrete examples are provided in the exploitation of self-nanoemulsifying systems, polymeric, lipid, and protein-based nanomedicines in offer pharmacokinetic-tailored approaches to improve the efficacy of drugs, particularly those with biopharmaceutics issues as vaccines. Insights on microfluidics scale-up production and microneedle-assisted administration of nanosystems are also approached in several contributions. Of special note, with honor of cover of this Issue, a study of albumin-based nanoparticles to promote the delivery of bevacizumab to colorectal cancer, by the group of Juan Irache.

Our Focus Group members are at the forefront of knowledge and innovation in the field of nanomedicines, and we are very proud to help them in disseminating their achievements and critical views. We hope that not only researchers involved in nanomedicines for drug delivery but also the general readers of Drug Delivery and Translational Research may find in this Issue relevant information for their current and future work.

\section{References}

1. RitaBosetti S, Jones L. Cost-effectiveness of nanomedicine: estimating the real size of nano-costs. Nanomedicine. 2019;14:1367-70.

2. Little SR. The current status and future directions of CRS Focus Groups. J Control Release. 2019;300:46-51.

Publisher's note Springer Nature remains neutral with regard to jurisdictional claims in published maps and institutional affiliations. 